\title{
La suplementación con zinc reduce la concentración de ácidos grasos libres en pacientes con diabetes tipo 2
}

\section{Zinc supplementation reduces free fatty acid concentration in patients with type 2 diabetes}

\section{RESUMEN}

Elevadas concentraciones de ácidos grasos libres (AGL) han sido asociadas con la patogénesis de resistencia a la insulina y diabetes tipo 2 (DT2), por lo que la regulación de la lipólisis resulta prioritaria en estos pacientes. El zinc mediante sus acciones insulinomiméticas e inducción de fosfodiesterasas podría regular la liberación de AGL desde el tejido adiposo. El objetivo de esta investigación fue evaluar en pacientes con DT2 el efecto de 24 meses de suplementación con zinc sobre las concentraciones séricas de AGL en ayuno. Para este propósito: se realizó la determinación de AGL en ayuno por colorimetría enzimática, zinc plasmático por espectrofotometría de absorción atómica, y parámetros bioquímicos y antropométricos de interés en 60 pacientes con DT2 que fueron asignados aleatoriamente para recibir suplementación con $30 \mathrm{mg} /$ día de zinc $(n=30)$ o placebo $(n=30)$ por 24 meses. El grupo zinc presentó menor concentración sérica de $A G L$ al mes $24(p=0,034)$. El cambio en el índice de masa corporal, el sexo y la suplementación con zinc contribuyeron significativamente como predictores de la concentración sérica de $A G L$ al mes $24\left(R=0,493, R^{2}=0,243, p=0,001\right)$. Conclusión: la suplementación con $30 \mathrm{mg} /$ día de zinc en pacientes con DT2 tuvo un efecto significativo en reducir la concentración sérica de AGL en ayuno tras 24 meses de tratamiento. Estos resultados apoyan los beneficios del zinc como coadyuvante en el tratamiento de DT2.

Palabras clave: Ácidos grasos libres; Diabetes tipo 2; Obesidad; Suplementación; Zinc.

\footnotetext{
ABSTRACT

High concentrations of free fatty acids (FFA) have been associated with the pathogenesis of insulin resistance and type 2 diabetes (T2D), making lipolysis regulation a priority in these patients. Through its insulin-mimetic actions and phosphodiesterase induction, zinc could regulate FFA release from adipose tissue. The objective of this research was to evaluate the effect of 24 months of zinc supplementation on fasting serum FFA concentrations in patients with T2D. For this purpose: fasting FFA by enzymatic colorimetric, plasma zinc by atomic absorption spectrophotometry, and
}

\begin{abstract}
María Catalina Hernández ${ }^{1 *}$, Pamela Rojas ${ }^{1}$, Fernando Carrasco ${ }^{1}$, Karen Basfi-fer ${ }^{1}$, Juana Codoceo ${ }^{1}$, Jorge Inostroza', Manuel Ruz'1.
\end{abstract}

1. Departamento de Nutrición, Facultad de Medicina, Universidad de Chile, Santiago, Chile.

*Dirigir correspondencia: María Catalina Hernández,
Departamento de Nutrición, Facultad de Medicina,
Universidad de Chile,
Independencia 1027, Santiago, Chile. E mail: mcatalina.hernandez@uchile.cl

Este trabajo fue recibido el 29 de julio de 2020. Aceptado con modificaciones: 28 de agosto de 2020. Aceptado para ser publicado: 09 de septiembre de 2020.

biochemical and anthropometric parameter of interest were determined in 60 T2D patients who were randomly assigned to $30 \mathrm{mg} /$ day of zinc supplementation $(n=30)$ or placebo $(n=30)$ for 24 months. The zinc group had lower serum FFA concentration at month $24(p=0.034)$. Body mass index change, gender, and zinc supplementation contributed significantly as predictors of serum FFA concentration at month $24\left(R=0.493, R^{2}=0.243, p=0.001\right)$. Conclusion: Supplementation with $30 \mathrm{mg} /$ day of zinc in patients with T2D had a significant effect in reducing serum fasting FFA concentration after 24 months of treatment. These results support the benefits of zinc as coadjutant in T2D treatment. Keywords: Free fatty acids; Obesity; Supplementation; Type 2 diabetes; Zinc. 


\section{INTRODUCCIÓN}

Los ácidos grasos libres (AGL) circulan en el torrente sanguíneo unidos predominantemente a albúmina ${ }^{1}$ y son el principal sustrato energético para el músculo esquelético, la corteza renal, el hígado y el miocardio². Sin embargo, elevadas concentraciones de AGL han sido asociadas con inflamación, disfunción endotelial, enfermedad cardiovascular, hipertensión, obesidad, síndrome metabólico ${ }^{3}$ y están involucradas directamente en la patogénesis de resistencia a la insulina ${ }^{4}$ y diabetes tipo 2 (DT2) ${ }^{5}$.

Los AGL están compuestos principalmente por ácidos grasos de cadena larga movilizados desde las reservas de triglicéridos (TG) en el tejido adiposo a través de la hidrólisis enzimática ${ }^{6}$. La lipólisis es un proceso que se encuentra finamente regulado. Las catecolaminas regulan positivamente la lipólisis mediante su unión a $\beta$-adrenoreceptores que al estar acoplados a proteína Gs llevan a la activación de la adenilato ciclasa, aumento en la producción de AMP cíclico (AMPC), activación de la proteína kinasa A (PKA), y consecuente fosforilación y activación de la perilipina y lipasa sensible a hormona (LSH); enzima que cataliza la hidrólisis de TG y liberación de ácidos grasos ${ }^{7}$. Por el contrario, la insulina es el principal regulador negativo de la lipólisis, mediante la unión a su receptor e inducción de la cascada de señalización río abajo, lleva a la activación de la fosfodiesterasa 3B (PDE3B); enzima que cataliza la conversión de AMPc a 5'-AMP, llevando a la reducción en la concentración intracelular de AMPc y consecuente supresión en la actividad de PKA y lipólisis ${ }^{8}$.

La DT2 es considerada en la actualidad como una enfermedad multifactorial compleja y el incremento en la lipólisis es uno de los componentes implicados en su patogénesis ${ }^{9}$, mediante mecanismos que incluyen la inducción de la resistencia a la insulina ${ }^{4}$, gluconeogénesis hepática ${ }^{10}$, hipertrigliceridemia ${ }^{11}$ y del estado proinflamatorio ${ }^{12}$. En adultos, una mayor concentración de AGL ha sido asociada con mayor incidencia de intolerancia a la glucosa y DT2 $2^{13}$ y en pacientes con DT2 han sido reportadas mayores concentraciones de $\mathrm{AGL}^{5}$. La regulación en la concentración de AGL circulantes resulta prioritario en pacientes con DT2, por lo cual, algunas investigaciones han evaluado el rol de agentes insulinomiméticos sobre la inhibición en la señalización de la lipólisis ${ }^{14}$. Al respecto, el zinc es un mineral esencial que ha demostrado ser capaz de inhibir a proteínas tirosina fosfatasas (PTPs) y activar a la PDE3B favoreciendo la vía de señalización de insulina, captación de glucosa mediada por insulina y atenuación en la fosforilación de LSH dependiente de PKA ${ }^{14,15}$. Por lo tanto, el zinc podría actuar como un regular negativo de la lipólisis capaz de simular la acción de la insulina sobre el tejido adiposo. Además, el zinc al igual que los AGL es transportado principalmente por albúmina ${ }^{16}$ y su fracción unida a albúmina compone la mayor parte del pool de zinc intercambiable en plasma'. Un incremento en la unión de AGL a albúmina lleva a la disrupción alostérica del sitio de unión de zinc, con un efecto deletéreo sobre la captación celular y estado nutricional de zinc ${ }^{1}$. Basados en la evidencia disponible planteamos que el tratamiento con zinc en pacientes con DT2 podría llevar a una reducción en las concentraciones séricas de AGL en ayuno, y esto se asociaría con el estado nutricional de zinc. El objetivo de esta investigación fue evaluar en pacientes con DT2 el efecto de 24 meses de suplementación con zinc sobre las concentraciones séricas de AGL en ayuno.

\section{MATERIALES Y MÉTODOS Participantes}

Este estudio fue desarrollado en una submuestra de 60 pacientes con DT2 que participaron en un ensayo clínico de suplementación con zinc por un periodo de 24 meses que buscaba evaluar el efecto del zinc sobre el control metabólico y evolución de la enfermedad (Proyecto DAZ, registrado en ISRCTN como ISRCTN95262434). Una descripción detallada del protocolo experimental se encuentra en la publicación de Pérez et al..17. Concisamente, los criterios de inclusión del ensayo fueron una hemoglobina glicosilada (HbA1c) menor a 9,0\%, glicemia en ayunas menor que $180 \mathrm{mg} / \mathrm{dL}$, edad entre 30 y 65 años, menos de 10 años de diagnóstico de la DT2, índice de masa corporal (IMC) entre 20 y 40 $\mathrm{kg} / \mathrm{m}^{2}$, y variación del peso corporal menor al $5,0 \%$ en los últimos 3 meses. Los criterios de exclusión fueron el uso de insulina, historia reciente de cetoacidosis diabética, falla renal, hepática y cardiaca, hipertensión, infarto, cáncer, infección aguda y cirugía, pacientes VIH positivo, gestantes o lactantes, y el uso de antipsicóticos, glucocorticoides y suplementos de vitaminas y/o minerales. El ensayo clínico original incluyó 80 participantes que fueron asignados aleatoriamente para recibir zinc (una cápsula diaria que contenía $30 \mathrm{mg}$ de zinc elemental como sulfato) o placebo (una cápsula diaria de silicato de magnesio) por un periodo de 24 meses, con determinaciones clínicas y bioquímicas al ingreso, 12 y 24 meses de tratamiento.

Los análisis para esta investigación se realizaron a partir de 30 muestras de sangre del grupo placebo y 30 del grupo zinc, tomadas aleatoriamente desde el conjunto de muestras del ensayo clínico original. Para las 60 muestras seleccionadas se realizaron las determinaciones de AGL séricos, zinc plasmático y parámetros bioquímicos. La información demográfica y antropométrica se obtuvo desde los registros del estudio original.

\section{Determinaciones}

La evaluación antropométrica se realizó mediante la determinación del peso, estatura y circunferencia de cintura (CC) a través de métodos estandarizados ${ }^{18}$. El IMC fue calculado $\left(\mathrm{kg} / \mathrm{m}^{2}\right)$ y categorizado en normalidad $\left(18,5-24,9 \mathrm{~kg} / \mathrm{m}^{2}\right)$; sobrepeso $\left(25,0-29,9 \mathrm{~kg} / \mathrm{m}^{2}\right)$ y obesidad $\left(\geq 30,0 \mathrm{~kg} / \mathrm{m}^{2}\right)$ según los puntos de corte establecidos por la Organización Mundial de la Salud $(\mathrm{OMS})^{19}$. Respecto a la composición corporal, de las regiones de interés (ROI) androide o abdominal y ginoide o glúteofemoral obtenidas mediante absorciometría de rayos $\mathrm{X}$ de doble 
energía (DXA) con el uso de un densitómetro Lunar DPX-L (Lunar Corporation, Madison, WI, USA, versión 1.30), se calculó la razón grasa androide/ginoide. La masa grasa total también fue obtenida por DXA. La insulina fue determinada por radioinmunoensayo (Siemens Healthcare Diagnostics Inc., Los Angeles, CA, USA). La glucosa, colesterol total, colesterol de $\mathrm{HDL}$, y triglicéridos fueron determinados por colorimetría de punto final (DIALAB GmbH, Wr. Neudorf, Austria), y se calculó el colesterol de LDL con la fórmula de Friedewald (colesterol LDL $(\mathrm{mg} / \mathrm{dL})=$ colesterol total $(\mathrm{mg} /$ $\mathrm{dL}$ ) - colesterol HDL $(\mathrm{mg} / \mathrm{dL})$ - triglicéridos $(\mathrm{mg} / \mathrm{dL}) / 5)^{20}$. El modelo homeostático para evaluar la resistencia a la insulina (HOMA-IR) se obtuvo aplicando la fórmula: HOMA-IR = insulina en ayunas $(\mu \mathrm{U} / \mathrm{mL}) \times$ glucosa en sangre en ayunas $(\mathrm{mg} / \mathrm{dL}) / 405^{21}$.

Muestras de suero obtenidas en condición de ayuno fueron empleadas para la determinación de la concentración de AGL mediante un método enzimático colorimétrico (NEFAHR (2); FUJIFILM Wako Diagnostics, Richmond, Virginia) siguiendo indicaciones del fabricante. El estándar NEFA fue empleado para la calibración, los resultados estuvieron dentro de los valores esperados indicados por el fabricante. El zinc plasmático fue determinado por espectrofotometría de absorción atómica siguiendo la metodología descrita por Smith et al. ${ }^{22}$ en un espectrofotómetro Perkin-Elmer AAnalyst 100 (Perkin-Elmer Corp., Waltham, MA, USA). El control de calidad del análisis de zinc se realizó utilizando Humatrol $\mathrm{N}$ como estándar (Human Gesellsachaft fur Biochemica and Diagnostica $\mathrm{MbH}$, Wiesbaden, Alemania).

\section{Análisis estadístico}

La prueba de Shapiro-Wilk fue empleada para evaluar si las variables seguían una distribución normal. Los valores se expresaron como n (\%) para variables categóricas, media \pm SD para variables que siguieron una distribución normal y promedio geométrico (IC 95\%) del antilogaritmo para las variables que alcanzaron una distribución normal luego de su transformación logarítmica. Las diferencias entre grupos fueron evaluadas mediante la prueba chi cuadrado $\left(X^{2}\right)$ y la prueba $t$ de Student para muestras independientes. Un ANOVA de dos factores con medidas repetidas en un factor fue empleado para evaluar el efecto del tiempo, del tratamiento y la interacción entre el tiempo y el tratamiento. Las asociaciones fueron evaluadas mediante el coeficiente de correlación de Pearson, además se realizó un análisis de regresión lineal múltiple para determinar las variables que tenían un efecto sobre la concentración sérica de AGL. Un valor de $\mathrm{p}<0,05$ fue considerado estadísticamente significativo.

\section{Aprobación ética}

Este estudio se realizó en conformidad con la Declaración de Helsinki de 1975, revisada en 2008. El protocolo de estudio fue aprobado por el Comité de Ética de Investigación en Seres Humanos de la Facultad de Medicina de la Universidad de Chile. Para cada uno de los participantes incluidos en la investigación se obtuvo el consentimiento informado.

\section{RESULTADOS}

En la tabla 1 se presentan las características basales de los grupos experimentales. No hubo diferencias por sexo y edad entre el grupo zinc y placebo. Con relación a la evaluación antropométrica, el grupo zinc presentó mayor IMC $(p=0,009)$ y circunferencia de cintura $(p=0,042)$; el $93,4 \%$ de los pacientes en el grupo zinc tenían exceso de peso en comparación con el 76,5\% en el grupo placebo. Respecto a la composición corporal, la grasa ginoide fue mayor en el grupo zinc $(p=0,020)$, y consecuentemente la razón androide/ginoide menor $(p=0,019)$, para la grasa androide y la masa grasa no hubo diferencias entre grupos. A los 24 meses, el IMC fue de 30,4 \pm 3,6 para el grupo zinc y de $27,6 \pm 3,8$ para el grupo placebo, y no hubo una interacción significativa entre el tiempo y el tratamiento para este parámetro.

Respecto a los parámetros bioquímicos basales no se encontraron diferencias entre grupos (Tabla 1). En conjunto para el grupo zinc y placebo, el 43,3\% de los voluntarios cumplía con la meta de glucosa en ayunas $(<130 \mathrm{mg} / \mathrm{dL})$ y el $73,8 \%$ con la de HbA1c $(<7,0 \%)$ establecida por la Asociación Americana de Diabetes $(\mathrm{ADA})^{23}$, la prevalencia de insulina en ayunas mayor al punto de corte $(\geq 15,0 \mu \mathrm{U} /$ $\mathrm{mL}$ ) fue del $13,3 \%$ y el índice HOMA-IR fue mayor que el punto de corte $(>2,6)$ en el $55 \%$ de los voluntarios, según los estándares establecidos para población general en Chile en el II Consenso de la Sociedad Chilena de Endocrinología y Diabetes sobre resistencia a la insulina ${ }^{24}$. El 78,3\% de los voluntarios presentó un nivel plasmático de colesterol total deseable $(<200 \mathrm{mg} / \mathrm{dL})$, y el $41,7 \%$ un nivel óptimo de colesterol LDL $(<100 \mathrm{mg} / \mathrm{dL})$ según los puntos de corte establecidos por el Programa Nacional de Educación sobre el Colesterol y el Panel III de Tratamiento del Adulto (ATPIII) ${ }^{25}$. Para el colesterol HDL, solo el 31,5\% de los hombres y el $12,0 \%$ de las mujeres presentó un nivel óptimo $(\geq 40 \mathrm{mg} / \mathrm{dL}$ $y \geq 50 \mathrm{mg} / \mathrm{dL}$, respectivamente), y para los triglicéridos, en el $41,7 \%$ de los voluntarios estuvo dentro del deseable $<150$ mg/dL) según los estándares 2020 de atención medica en diabetes de la $\mathrm{ADA}^{26}$.

En la figura 1 se presentan las concentraciones séricas de AGL en ayuno. Se encontró una interacción significativa entre el tiempo y el tratamiento, con menor concentración de AGL en suero en el grupo zinc al mes 24 de tratamiento (comparaciones múltiples ajustadas por Bonferroni: $p=0,034)$.

En la figura 2 se presentan las concentraciones plasmáticas de zinc. Se encontró una interacción significativa entre el tiempo y el tratamiento, con mayor concentración de zinc en plasma para el grupo zinc al mes 12 de tratamiento, pero no al mes 24 (comparaciones múltiples ajustadas por Bonferroni: $p=0,001$ y $p=0,803$, respectivamente).

En la figura 3 se presenta la asociación entre el zinc plasmático y los AGL circulantes antes de iniciar la suplementación con zinc. No se encontró una correlación significativa entre la concentración plasmática de zinc y la concentración sérica de AGL en ayuno $(r=-0,142, p=0,280)$. 
Se realizaron análisis de correlación bivariados como un primer acercamiento para conocer la potencial asociación entre variables, y posteriormente, proceder con un análisis de regresión lineal múltiple utilizando como variable dependiente la concentración sérica de AGL al mes 24 . Inicialmente, se efectuó un análisis que contemplaba las siguientes variables = sexo, cambio en el IMC (mes 24-mes $0)$, suplementación con zinc, cambio en la concentración de zinc plasmático (mes 24-mes 0), y cambio de la relación androide/ginoide (mes 24-mes 0). El cambio en la CC se excluyó del análisis por presentar colinealidad con variables independientes. Posteriormente se removieron aquellas variables que presentaron un valor de coeficiente B no significativo, generándose el modelo que se presenta en la tabla 2, a través del cual se determinó que finalmente el cambio en el IMC, el sexo y la suplementación con zinc contribuían significativamente como predictores de la concentración sérica de AGL al mes 24 , y en conjunto explican en un $24,3 \%$ el cambio en la concentración de AGL circulantes.

Tabla 1. Parámetros basales en pacientes con diabetes tipo 2 suplementados con zinc o placebo durante 24 meses.

\begin{tabular}{|c|c|c|c|}
\hline Parámetros & Grupo placebo & Grupo zinc & $\mathbf{p}$ \\
\hline \multicolumn{4}{|l|}{ Demográficos } \\
\hline Hombres & $19(63,3)$ & $16(53,3)$ & $0,432^{\mathrm{a}}$ \\
\hline Mujeres & $11(36,7)$ & $14(46,7)$ & \\
\hline Edad (años) & $54,9 \pm 6,40$ & $55,0 \pm 7,56$ & $0,985^{b}$ \\
\hline \multicolumn{4}{|l|}{ Antropométricos } \\
\hline Estatura $(\mathrm{m})$ & $1,65 \pm 0,09$ & $1,64 \pm 0,11$ & $0,547^{b}$ \\
\hline Peso $(k g)$ & $75,9 \pm 12,8$ & $81,3 \pm 15,6$ & $0,150^{\mathrm{b}}$ \\
\hline $\mathrm{IMC}\left(\mathrm{kg} / \mathrm{m}^{2}\right)$ & $27,7 \pm 3,35$ & $30,1 \pm 3,59$ & $0,009^{b}$ \\
\hline Clasificación por IMC: normalidad & $7(23,3)$ & $2(6,7)$ & $0,108^{a}$ \\
\hline sobrepeso & $15(50,0)$ & $14(46,7)$ & \\
\hline obesidad & $8(26,7)$ & $14(46,7)$ & \\
\hline $\mathrm{CC}(\mathrm{cm})$ & $94,6 \pm 8,70$ & $99,4 \pm 9,05$ & $0,042^{\mathrm{b}}$ \\
\hline \multicolumn{4}{|l|}{ Composición corporal } \\
\hline Masa grasa $(\%)$ & $21,7(19,9-26,2)$ & $25,6(23,6-30,0)$ & $0,094^{b}$ \\
\hline Grasa androide (\%) & $32,2 \pm 7,12$ & $35,3 \pm 7,48$ & $0,120^{\mathrm{b}}$ \\
\hline Grasa ginoide (\%) & $25,4 \pm 7,69$ & $30,7 \pm 8,84$ & $0,020^{b}$ \\
\hline Razón androide/ginoide & $1,31 \pm 0,22$ & $1,18 \pm 0,18$ & $0,019^{b}$ \\
\hline \multicolumn{4}{|l|}{ Bioquímicos } \\
\hline Glucosa en ayunas (mg/dL) & $133 \pm 21,7$ & $132 \pm 21,6$ & $0,932^{\mathrm{b}}$ \\
\hline Insulina en ayunas $(\mu \mathrm{U} / \mathrm{mL})$ & $7,60(6,94-9,99)$ & $9,21(8,31-13,0)$ & $0,149^{b}$ \\
\hline Índice HOMA-IR & $2,45(2,25-3,29)$ & $2,96(2,70-4,19)$ & $0,181^{b}$ \\
\hline HbA1c (\%) & $6,5(6,3-6,8)$ & $6,3(6,0-6,6)$ & $0,249^{b}$ \\
\hline Colesterol total (mg/dL) & $176 \pm 32,9$ & $171 \pm 33,2$ & $0,586^{b}$ \\
\hline Colesterol HDL (mg/dL) & $37,8 \pm 9,6$ & $36,9 \pm 10,9$ & $0,753^{b}$ \\
\hline Colesterol LDL (mg/dL) & $109 \pm 27,5$ & $107 \pm 32,2$ & $0,857^{\mathrm{b}}$ \\
\hline Triglicéridos (mg/dL) & $134(121-177)$ & $128(118-156)$ & $0,703^{\mathrm{b}}$ \\
\hline
\end{tabular}

Valores son expresados como n (\%) para variables categóricas, media \pm SD para variables que siguieron una distribución normal y promedio geométrico (IC 95\%) del antilogaritmo para variables que alcanzaron una distribución normal luego de su transformación logarítmica, $\mathrm{n}=30$ por grupo. ${ }^{\mathrm{a}} \mathrm{X}^{2}$, b prueba t de Student para muestras independientes. IMC: índice de masa corporal; CC: circunferencia de cintura. 


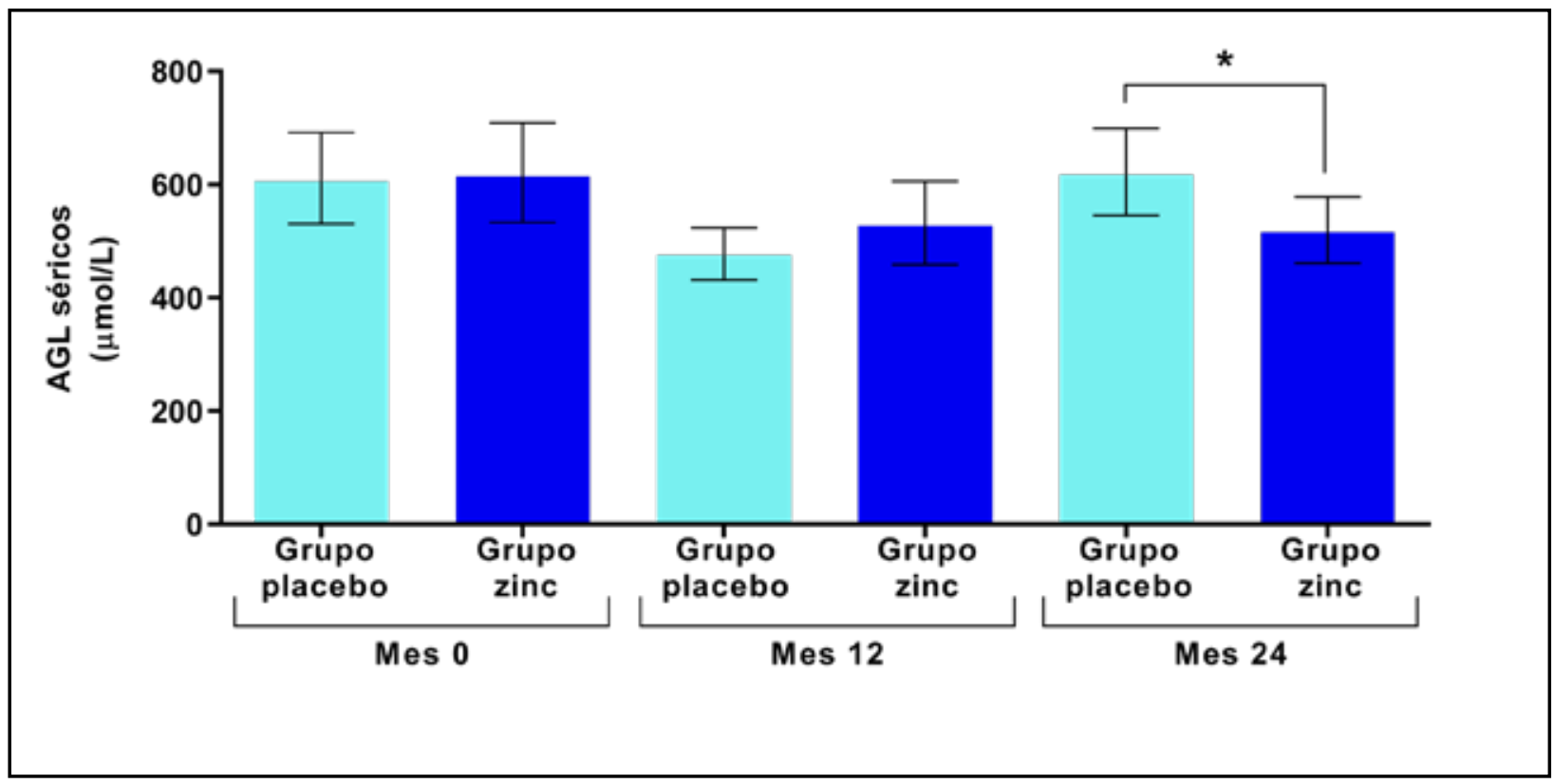

Figura 1: Concentraciones séricas de ácidos grasos libres en ayuno de pacientes con diabetes tipo 2 suplementados con zinc o placebo por 24 meses. Las barras representan la media geométrica (IC 95\%) del antilogaritmo de la variable al alcanzar la distribución normal luego de su transformación logarítmica, $n=30$ por grupo. * ANOVA de dos factores con medidas repetidas en un factor seguido de comparaciones múltiples ajustadas por Bonferroni, interacción: Tiempo x Tratamiento, $F(1,58)=4.739, p=0,034$. AGL: ácidos grasos libres.

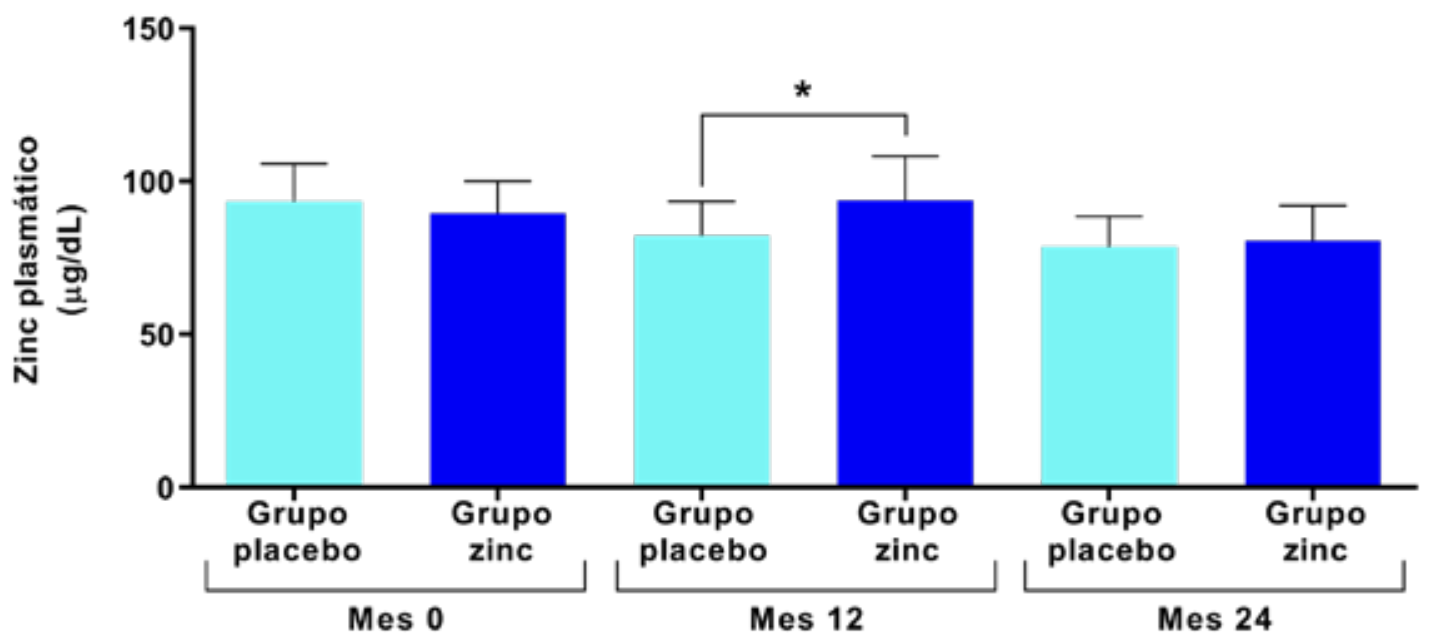

Figura 2: Concentraciones plasmáticas de zinc en pacientes con diabetes tipo 2 suplementados con zinc o placebo por 24 meses. Las barras representan la media $\pm \mathrm{SD}, \mathrm{n}=30$ por grupo. * ANOVA de dos factores con medidas repetidas en un factor seguido de comparaciones múltiples ajustadas por Bonferroni, interacción: Tiempo x Tratamiento, F (1, 58) = 15.178, $p=0,000$. 


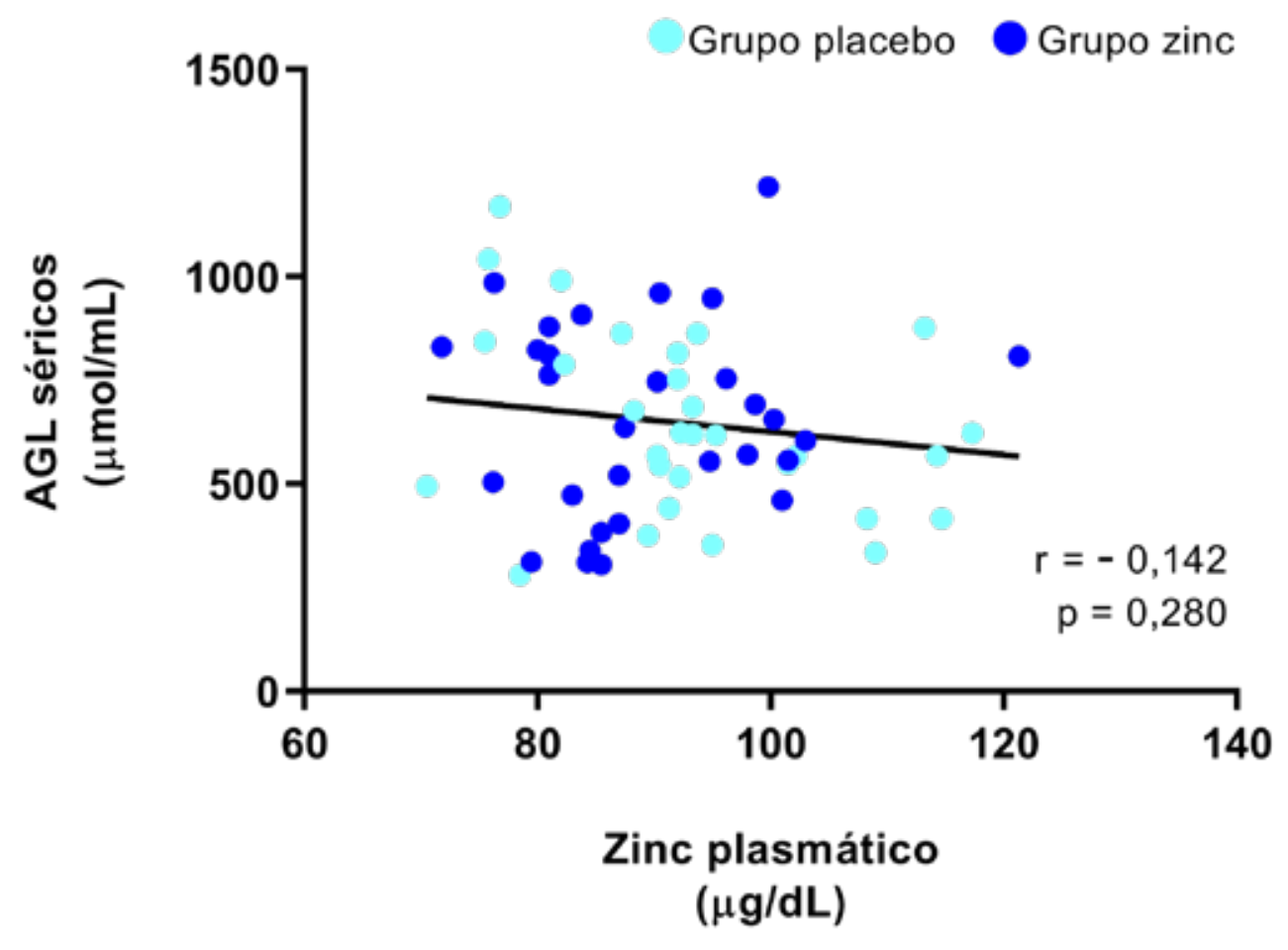

Figura 3: Asociación entre la concentración plasmática de zinc y la concentración sérica de ácidos grasos libres en ayuno de pacientes con diabetes tipo 2 antes de recibir los tratamientos con zinc o placebo. El gráfico representa el diagrama de dispersión entre la concentración basal de zinc y la concentración sérica de AGL. $\mathrm{n}=60$ (30 por grupo). r= coeficiente de correlación de Pearson. AGL: ácidos grasos libres.

Tabla 2. Análisis de correlación múltiple entre la concentración de ácidos grasos libres frente al cambio en el IMC, el sexo y la suplementación con zinc.

\section{$\mathrm{Y}($ concentración sérica de AGL al mes 24) =}

\begin{tabular}{|c|c|c|}
\hline & B & $\mathbf{p}$ \\
\hline Cambio en el IMC (mes $24-$ mes 0) & 43,3 & 0,036 \\
\hline Sexo (0 femenino; 1 masculino) & $-115,7$ & 0,019 \\
\hline $\mathrm{R}=0,493, \mathrm{R}^{2}=0,243$, ANOVA: $\mathrm{F}(3,5$ & $\mathrm{p}=0,001$ & \\
\hline
\end{tabular}

Predictores de la concentración de ácidos grasos libres (AGL) en ayuno al mes 24 en un modelo de regresión lineal múltiple: (Constante), cambio en el IMC, sexo, suplementación con zinc. $n=60$. IMC: índice de masa corporal. 


\section{DISCUSIÓN}

El potencial rol del zinc como un agente regulador negativo de la lipólisis surgió a partir de observaciones en modelo animal en que en ratas con deficiencia de zinc, las concentraciones de AGL circulantes bajo condición de ayuno eran casi el doble que en los controles, y esto se atribuía a una mayor degradación de las reservas de TG en tejido adiposo ${ }^{27}$. Además, los mecanismos fueron apoyados por la acción insulinomimética del zinc y su impacto en el metabolismo de la glucosa, dado que en ratas con deficiencia de zinc también se observaba una reducción en la incorporación de glucosa en tejido graso epididimal ${ }^{28}$.

Una desregulación en la lipólisis y consecuentemente un incremento en los AGL circulantes ha sido asociado como factor etiológico de múltiples condiciones patológicas", razón por la cual diversos estudios han buscado demostrar la eficacia del tratamiento con zinc como opción terapéutica enfocada a regular las tasas de lipólisis. Estudios in vitro han demostrado que en adipocitos 3T3-L1 el tratamiento con complejos de zinc-alicina y sus derivados, suprime la fosforilación de la LSH mediada por la actividad de la PKA dependiente de AMPc, Ilevando a inhibición en la liberación de AGL, y además, mejora la captación de glucosa dependiente de la translocación de GLUT4 a la membrana, mediante inducción de la fosforilación de la proteína quinasa $B(A K T / P K B)$, mejorando de este modo tanto la utilización de la glucosa como el metabolismo de lípidos ${ }^{14}$. In vivo el tratamiento con nanopartículas de óxido de zinc en ratas diabéticas ha demostrado reducir en un $40 \%$ las concentraciones circulantes de AGL e inducir una mejoría en la tolerancia a la glucosa con reducción en la glucosa sanguínea e incremento en la secreción de insulina $^{15}$. Sin embargo, son escasos los ensayos clínicos que han evaluado el efecto de la suplementación con zinc sobre la lipólisis y más aun bajo una condición de DT2, en la cual el déficit en la acción y secreción de insulina que subyace a la enfermedad favorece las tasas de lipólisis. Hasta donde conocemos, este es el primer estudio en pacientes con DT2 que evalúa el efecto de la suplementación crónica con zinc sobre las concentraciones de AGL en ayuno, demostrando que el tratamiento con $30 \mathrm{mg} /$ día de zinc elemental efectivamente reduce la concentración de AGL en ayuno.

Interesantemente, el tratamiento con zinc tuvo un efecto en reducir la concentración de AGL en ayuno en el grupo zinc a pesar de presentar condiciones basales de mayor obesidad y circunferencia de cintura que el grupo control. Los pacientes con obesidad presentan mayores concentraciones de AGL debido a que la masa de tejido adiposo se encuentra aumentada y por ende su capacidad de liberar AGL, el aclaramiento de AGL disminuido, y a su vez los AGL circulantes inhiben la acción antilipolítica de la insulina, aumentando aún más la tasa de liberación de AGL a circulación ${ }^{29}$. Además, la circunferencia de cintura se correlaciona directamente con la concentración de $\mathrm{AGL}^{30}$. La distribución topográfica de la grasa corporal también se ha asociado diferencialmente con el riesgo de resistencia a la insulina y diabetes. La obesidad androide implica un mayor riesgo que la ginoide, debido a que la primera involucra un mayor contenido de tejido adiposo visceral, con mayor actividad y expresión génica de $\beta$-adrenoreceptores implicados en la lipólisis ${ }^{31}$. En este estudio no se encontraron diferencias respecto al \% de grasa androide que puedan explicar las concentraciones de AGL, y el cambio en la relación androide/ginoide no fue predictor de la concentración sérica de AGL al mes 24, Por el contrario, para el IMC el cambio entre el mes 24 y el basal si influyó significativamente en la concentración sérica de AGL al mes 24, aun cuando la suplementación con zinc no tuvo un efecto sobre el IMC.

El zinc es esencial para el correcto procesamiento, almacenamiento y secreción de insulina, además, ejerce acciones insulinomiméticas principalmente al inhibir a la proteína tirosina fosfatasa 1B (PTP1B), facilitando así la fosforilación neta del receptor de insulina, y mediante la activación de componentes claves en la vía de señalización de insulina como lo son las quinasas reguladas por señales extracelulares $1 / 2$ (ERK 1/2) y la fosfatidilinositol 3-quinasa / proteína quinasa $\mathrm{B}(\mathrm{PI} 3-\mathrm{K} / \mathrm{PKB})^{32}$. En cuanto al rol específico de este nutriente sobre los AGL, el posible mecanismo a través del cual el zinc estaría ejerciendo el efecto observado sobre la regulación en la concentración sérica de AGL en ayuno podría atribuirse principalmente a la función de este mineral como regulador negativo de la lipólisis mediante su capacidad de inhibir PTPs e inducir la actividad enzimática de la PDE3B a través de la vía PI3-K/PKB, con la consecuente reducción en los niveles intracelulares de AMPc, atenuación en la fosforilación de la LSH dependiente de PKA y finalmente, reducción en la liberación de AGL desde el tejido adiposo ${ }^{14,15}$. Así, la suplementación con zinc podría estar favoreciendo tanto la secreción como la disponibilidad de insulina en el tejido adiposo, permitiendo de este modo que la insulina pueda ejercer su rol como principal regulador negativo de la lipólisis ${ }^{8}$, y además, favoreciendo la cascada de señalización de insulina en el adipocito, con una consecuente inhibición de la lipólisis mediada principalmente por la activación de la PDE3B. Por otra parte, dado que la concentración de AGL circulantes también se encuentra determinada por la lipogénesis de novo y consecuente liberación de ácidos grasos al sistema venoso portal ${ }^{33}$; el zinc también podría estar actuando a través de su rol en el metabolismo de los ácidos grasos. $\mathrm{Al}$ respecto, el zinc como cofactor de las enzimas delta desaturasas favorece la síntesis endógena de ácidos grasos poliinsaturados ${ }^{34}, y$ ha sido demostrado que los ácidos grasos poliinsaturados de cadena larga de la línea n-3 disminuyen la lipogénesis de novo hepática a través de una reducción en la disponibilidad nuclear y regulación a la baja en la expresión del factor de transcripción lipogénico: proteína de unión al elemento regulador del esterol-1c (SREBP-1c) y de sus genes blanco ${ }^{35,36}$. Además, el zinc a través de sus mecanismos antioxidantes puede ejercer una protección 
contra reacciones de lipoperoxidación ${ }^{37}$ y así favorecer la integridad y disponibilidad de estos ácidos grasos para ejercer los efectos biológicos sobre SREBP-1c anteriormente mencionados. Estos aspectos requieren más investigaciones que evalúen específicamente el efecto del zinc sobre la concentración de AGL circulantes mediado por su impacto en el metabolismo de los ácidos grasos.

Como era de esperar, la suplementación con zinc tuvo un efecto positivo sobre el estado nutricional de zinc, con mayor concentración plasmática de zinc a los 12 meses de tratamiento. Sin embargo, este efecto no se observó a los 24 meses. Este fenómeno no es tan inusual, ya que si bien el zinc plasmático ha demostrado ser un biomarcador útil para evaluar la exposición a suplementos de zinc ${ }^{38}$, presenta limitaciones dado que no necesariamente refleja el estado celular de zinc debido a finos mecanismos de control homeostáticos, y el zinc plasmático muestra amplia variabilidad interindividual y respuesta diferencial a cambios en la dieta, además, de verse afectado por condiciones de estrés, infección o cambios hormonales ${ }^{38}$. Por otra parte, elevadas concentraciones de AGL circulantes también podrían tener un efecto negativo en la concentración de zinc circulante. Entre el $75-90 \%$ del zinc total en plasma se encuentra unido a albúmina, esta interacción zinc-albúmina facilita la captación de zinc por las células endoteliales ${ }^{39} y$ eritrocitos $^{40}$. Los AGL luego de ser liberados a la circulación desde reservas en tejido adiposo también son transportados por albúmina, así el incremento en la unión de AGL a albúmina lleva a interrupción del sitio de unión a zinc, alteración en la captación celular de zinc e hipozincemia ${ }^{1,2}$.

Finalmente, un elemento clave para la interpretación de los resultados es la alta adherencia que registraron los pacientes en cuanto al consumo de placebo o suplemento de zinc, la cual fue de $97,0 \%$, reflejando esta cifra la proporción de cápsulas efectivamente consumidas en relación a lo planificado. Esto queda plasmado en el análisis de regresión múltiple que retiene solo las variables con un valor significativo del coeficiente $B$, el cual muestra que una persona de sexo masculino, que recibe suplementación con zinc y que tiene una menor ganancia de peso corporal (IMC), tendrá un valor menor de AGL al cabo de un periodo de suplementación de 24 meses.

\section{CONCLUSIÓN}

En esta investigación encontramos que la suplementación con $30 \mathrm{mg} / \mathrm{día}$ de zinc elemental en pacientes con DT2 tuvo un efecto en reducir la concentración sérica de AGL en ayuno tras 24 meses de tratamiento, además, el cambio en el IMC y el sexo contribuyeron significativamente como predictores de la concentración sérica de AGL al mes 24, indicando que pacientes con DT2 de sexo masculino, que presentan una menor ganancia de peso corporal y reciben suplementación con zinc, tendrán una menor concentración de AGL tras 24 meses de tratamiento. Estos resultados apoyan los beneficios del zinc como un coadyuvante en el tratamiento de la DT2.
Financiamiento: Esta investigación fue financiada por el Fondo Nacional de Desarrollo Científico y Tecnológico (FONDECYT), los proyectos de investigación 1120323 y 1160792, la Sociedad Chilena de Nutrición Bromatología y Toxicología (SOCHINUT), proyecto SCHINUT303, y la Agencia Nacional de Investigación y Desarrollo (ANID) / Programa de Becas / DOCTORADO NACIONAL / 2016 - 21160453 (a MCH).

\section{BIBLIOGRAFÍA}

1. Barnett JP, Blindauer CA, Kassaar O, Khazaipoul S, Martin EM, Sadler PJ, et al. Allosteric modulation of zinc speciation by fatty acids. Biochim Biophys Acta. 2013; 1830: 5456-5464.

2. Coppack SW, Jensen MD, Miles JM. In vivo regulation of lipolysis in humans. J Lipid Res. 1994; 35: 177-193.

3. Ghosh A, Gao L, Thakur A, Siu PM, Lai CWK. Role of free fatty acids in endothelial dysfunction. J Biomed Sci. 2017; 24: 1-15.

4. Boden G. Free fatty acids - The link between obesity and insulin resistance. Endocr Pract. 2001; 7: 44-51.

5. Kahn SE, Hull RL, Utzschneider KM. Mechanisms linking obesity to insulin resistance and type 2 diabetes. Nature. 2006; 444: 840-846.

6. Lafontan $M$, Langin D. Lipolysis and lipid mobilization in human adipose tissue. Prog Lipid Res. 2009; 48: 275-297.

7. Carmen G-Y, Víctor S-M. Signalling mechanisms regulating lipolysis. Cell Signal. 2006; 18: 401-408.

8. Kitamura T, Kitamura Y, Kuroda S, Hino Y, Ando M, Kotani K, et al. Insulin-induced phosphorylation and activation of cyclic nucleotide phosphodiesterase 3B by the serine-threonine kinase Akt. Mol Cell Biol. 1999; 19: 6286-6296.

9. Defronzo RA. Banting Lecture. From the triumvirate to the ominous octet: a new paradigm for the treatment of type 2 diabetes mellitus. Diabetes. 2009; 58: 773-795.

10. Chen X, Iqbal N, Boden G. The effects of free fatty acids on gluconeogenesis and glycogenolysis in normal subjects. J Clin Invest. 1999; 103: 365-372.

11. Julius U. Influence of plasma free fatty acids on lipoprotein synthesis and diabetic dyslipidemia. Exp Clin Endocrinol Diabetes. 2003; 111: 246-250.

12. Schenk S, Saberi M, Olefsky JM. Insulin sensitivity: modulation by nutrients and inflammation. J Clin Invest. 2008; 118: 29923002.

13. Salgin B, Ong KK, Thankamony A, Emmett P, Wareham NJ, Dunger $D B$. Higher fasting plasma free fatty acid levels are associated with lower insulin secretion in children and adults and a higher incidence of type 2 diabetes. I Clin Endocrinol Metab. 2012; 97: 3302-3309.

14. Hiromura M, Sakurai H. Action mechanism of metallo-allixin complexes as antidiabetic agents. Pure Appl Chem. 2008; 80: 2727-2733.

15. Umrani RD, Paknikar KM. Zinc oxide nanoparticles show antidiabetic activity in streptozotocin-induced Type 1 and 2 diabetic rats. Nanomedicine (Lond). 2014; 9: 89-104.

16. Smith $K T$, Failla ML, Cousins RJ. Identification of albumin as the plasma carrier for zinc absorption by perfused rat intestine. Biochem J. 1979; 184: 627-633.

17. Perez A, Rojas P, Carrasco F, Basfi-fer K, Perez-Bravo F, Codoceo J, et al. Association between zinc nutritional status and glycemic control in individuals with well-controlled type-2 diabetes. J Trace Elem Med Biol. 2018; 50: 560-565. 
18. Gibson, RS. Principles of Nutritional Assessment. Oxford University Press, New York, 2005.

19. World Health Organization. Obesity: Preventing and Managing the Global Epidemic: Report on a WHO Consultation (WHO Technical Report Series 894). Geneva, Switzerland: World Health Organization; 2000.

20. Friedewald WT, Levy RI, Fredrickson DS. Estimation of the concentration of low-density lipoprotein cholesterol in plasma, without use of the preparative ultracentrifuge. Clin Chem. 1972; 18: 499-502.

21. Matthews DR, Hosker JP, Rudenski AS, Naylor BA, Treacher $D F$, Turner RC. Homeostasis model assessment: insulin resistance and $\beta$-cell function from fasting plasma glucose and insulin concentrations in man. Diabetologia. 1985; 28: 412-419.

22. Smith JC, Butrimovitz GP, Purdy WC. Direct measurement of zinc in plasma by atomic absorption spectroscopy. Clin Chem. 1979; 25: 1487-1491.

23. American Diabetes Association. 6. Glycemic Targets: Standards of Medical Care in Diabetes-2020. Diabetes Care. 2020; 43: S66-S76.

24. Pollak F, Araya V, Lanas A, Sapunar J, Arrese M, Aylwin CG lori, et al. Second Consensus of the Chilean Society of Endocrinology and Diabetes about insulin resistance. Rev Med Chil. 2015; 143: 627-636.

25. Third Report of the National Cholesterol Education Program (NCEP) Expert Panel on Detection, Evaluation, and Treatment of High Blood Cholesterol in Adults (Adult Treatment Panel III) final report. Circulation. 2002; 106: 3143-3421.

26 American Diabetes Association. 10. Cardiovascular disease and risk management: standards of medical care in diabetes-2020. Diabetes Care. 2020; 43: S111-S134.

27. Quarterman J, Florence E. Observations on glucose tolerance and plasma levels of free fatty acids and insulin in the zincdeficient rat. Br J Nutr. 1972; 28: 75-79.

28. Reeves PG, O'Dell BL. The effect of zinc deficiency on glucose metabolism in meal-fed rats. Br / Nutr. 1983; 49: 441-452.

29. Jensen MD, Haymond MW, Rizza RA, Cryer PE, Miles JM. Influence of body fat distribution on free fatty acid metabolism in obesity. J Clin Invest. 1989; 83: 1168-1173.

30. Yang $R F$, Zhang $H$, Wang $Z$, Liu $X Y$, Lin Z. A study on the relationship between waist phenotype, hypertriglyceridemia, coronary artery lesions and serum free fatty acids in adult and elderly patients with coronary diseases. Immun Ageing. 2018; 15: 14.

31. Van Harmelen $V$, Lönnqvist $F$, Thörne A, Wennlund A, Large $V$, Reynisdottir $S$, et al. Noradrenaline-induced lipolysis in isolated mesenteric, omental and subcutaneous adipocytes from obese subjects. Int J Obes. 1997; 21: 972-979.

32. Vardatsikos G, Pandey NR, Srivastava AK. Insulino-mimetic and anti-diabetic effects of zinc. I Inorg Biochem. 2013; 120: 8-17.

33. Milić S, Lulić D, Štimac D. Non-alcoholic fatty liver disease and obesity: Biochemical, metabolic and clinical presentations. World J Gastroenterol. 2014; 20: 9330-9337.

34. Cunnane SC. Differential regulation of essential fatty acid metabolism to the prostaglandins: Possible basis for the interaction of zinc and copper in biological systems. Prog Lipid Res. 1982; 21: 73-90.

35. Valenzuela $R$, Videla $L A$. Impact of the co-administration of $n-3$ fatty acids and olive oil components in preclinical nonalcoholic fatty liver disease models: A mechanistic view. Nutrients. 2020; 12: 499.

36. Valenzuela $R$, Ortiz M, Hernández-Rodas MC, Echeverría $F$, Videla $L A$. Targeting $n-3$ polyunsaturated fatty acids in non-alcoholic fatty liver disease. Curr Med Chem. 2019; 26: $1-21$.

37. Fortes $C$, Agabiti N, Fano V, Pacifici R, Forastiere $F$, Virgili $F$, et al. Zinc supplementation and plasma lipid peroxides in an elderly population. Eur J Clin Nutr. 1997; 51: 97-101.

38. King JC, Brown KH, Gibson RS, Krebs NF, Lowe NM, Siekmann $\mathrm{JH}$, et al. Biomarkers of nutrition for development (BOND) - Zinc review. J Nutr. 2015; 146: 858S-885S.

39. Rowe DJ, Bobilya DJ. Albumin facilitates zinc acquisition by endothelial cells. Proc Soc Exp Biol Med. 2000; 224: 178-186.

40. Gálvez M, Moreno JA, Elósegui LM, Escanero JF. Zinc uptake by human erythrocytes with and without serum albumins in the medium. Biol Trace Elem Res. 2001; 84: 45-56. 